\title{
Trombosis de senos venosos cerebrales en menores de dos meses de edad y sus secuelas
}

\author{
Cerebral sinovenous thrombosis in children under two months old and sequels
}

Jorge Eduardo Ortega, * Ángel Federico Velásquez Castillo,* Lidia María Prado López.**

\section{RESUMEN}

Antecedentes: La trombosis de senos venosos cerebrales (TSVC) es un padecimiento infrecuente que puede tener alta mortalidad y secuelas neurológicas severas, por su variada sintomatología puede ser mal diagnosticada. Objetivo: Determinar la presentación clínica, laboratorial y tomográfica, así como las secuelas en niños menores de 2 meses de edad con trombosis de senos venosos cerebral atendidos en el Hospital Nacional Mario Catarino Rivas (HNMCR) de enero del 2009 a febrero del 2013. Pacientes y Métodos: estudio descriptivo con diseño longitudinal. La muestra fue de 14 niños menores de 2 meses de edad a quienes se diagnosticó TSVC y se obtuvieron datos clínicos, laboratoriales y tomográficos. Se les realizó seguimiento para determinar las secuelas psicomotoras. Resultados: las manifestaciones clínicas reportadas fueron fontanela tensa en todos los pacientes, convulsiones en el $86 \%$ y deshidratación en el $71 \%$. Todos presentaron anemia con niveles de hemoglobina promedio de $6.7 \mathrm{~g} / \mathrm{dl}$. La tomografía cerebral mostró afectación de los senos: sagital superior $100 \%$, lateral izquierdo $78.6 \%$, lateral derecho $71.4 \%$ y recto $50 \% .7$ niños tuvieron retraso psicomotor, 4 tuvieron desarrollo normal y 3 fallecieron. Conclusión: La TSVC sigue siendo una patología vascular poco comprendida y posiblemente sub diagnosticada que amerita mayor atención del cuerpo médico.

\section{PALABRAS CLAVE:}

Trombosis intracraneal, lactante, Tomografía.

* Neurocirujano Hospital Nacional Dr. Mario Catarino Rivas (HNMCR)

** Pediatra, HNMCR

Dirigir correspondencia a: neurortega@hotmail.com

Recibido: 15 de octubre 2015 Aprobado: 31 de marzo 2016

\section{ABSTRACT}

Background: cerebral venous thrombosis (CVT) is a rare condition with varied symptoms that can be misdiagnosed. It can have a high mortality rate and severe neurological sequelaes. Objective: To determine the clinical, laboratorial and tomographic presentation and sequelaes in children younger than 2 months old with cerebral venous thrombosis at the National Hospital Mario Catarino Rivas (HNMCR) from January 2009 to February 2013. Patients and Methods: a descriptive study with a longitudinal design was. The performed sample consisted of 14 children younger than 2 months of age who were diagnosed with. CVT Clinical, laboratory and tomographic data were obtained. They were followed up to determine psychomotor sequelaes. Results: The clinical manifestations reported were tense fontanelle in all patients, seizures in $86 \%$ and dehydration $71 \%$. All had anemia with average hemoglobin levels of $6.7 \mathrm{~g} / \mathrm{dl}$. The brain scan showed involvement of the following sinuses: superior sagittal $100 \%$, left side $78.6 \%$, right side $71.4 \%$ and straight sinus $50 \% .7$ children had severe psychomotor retardation, 4 had a normal development and 3 died. Conclusion: CVT remains a poorly understood and possibly sub diagnosed vascular pathology that deserves greater attention from the medical profession.

\section{KEYWORDS}

Intracraneal thrombosis, infant, tomography.

\section{INTRODUCCIÓN}

Las trombosis de senos venosos cerebrales es una enfermedad caracterizada por la coagulación espontánea de uno o más senos venosos y las venas que drenan el flujo sanguíneo cerebral produciendo una congestión venosa que 
se traduce en la presentación de síntomas inespecíficos, muy variados, que pueden ir desde irritabilidad hasta el coma, o incluso la muerte. ${ }^{(1)}$

La incidencia de la TSVC ha sido estimada en 2.6 casos por 100,000 neonatos por año. ${ }^{(1-3)}$ Se desconoce la incidencia a nivel local, muchos casos pasan desapercibidos y los que son detectados son por hallazgos incidentales al interconsultar con el servicio de neurocirugía por sospechas de hemorragias cerebrales. Por este motivo se plantea el objetivo de describir la presentación clínica, laboratorial y tomográficade la TSVC y sus secuelas en niños menores de dos meses en el HNMCR en el periodo comprendido entre enero del 2009 a febrero del 2013.

\section{PACIENTES Y MÉTODOS}

Se trata de un estudio descriptivo con diseño longitudinal. El universo lo conforman los pacientes que acudieron al servicio de emergencia de Pediatría y fueron presentados al Servicio de Neurocirugía del HNMCR con diagnósticos de hematoma subdural o intraparenquimatoso de etiología no determinada. La muestra la conformaron los pacientes a quienes se confirmó el diagnóstico de TSVC mediante TAC, en el período antes definido $(n=14)$.

Los criterios de inclusión fueron: niños con diagnóstico comprobado por tomografía de TSVC durante el periodo del estudio. No se excluyeron casos.

Se obtuvo el consentimiento de los tutores legales para participar en este estudio.Se recabaron los datos de los pacientes al momento de hacerse el diagnóstico de TSVC y se buscaron factores de morbilidad tales como deshidratación, sepsis o defectos congénitos.

Se obtuvieron los resultados de laboratorio de recuento leucocitario en $\mathrm{cel} / \mathrm{ml}$, hemoglobina en $\mathrm{g} / \mathrm{dl}$ y recuento plaquetario en $\mathrm{plt} / \mathrm{ml}$. Por limitaciones de laboratorio en ninguno de los pacientes se realizó estudios especiales de factores de riesgos protrombóticos. A partir de las placas de tomografía cerebral se determinaron los senos afectados. Se registraron las defunciones y en los sobrevivientes se les practicó al menos un estudio tomográfico 15 días después de realizado su primera tomografía y un seguimiento clínico entre 6 y 30 meses para determinar las secuelas neurológicas.

Para el análisis de datos se utilizó el programa EPI INFO 7.5.1.2 donde se calcularon medidas de frecuencia, tendencia central y medidas de disperción. Para la presentación de datos se utilizó el programa de Microsoft Office Excel 2007.

\section{RESULTADOS}

Entre los hallazgos demográficos se captaron 6 recién nacidos masculinos y 8 recién nacidos femeninos con una relación hombre:mujer de $1: 1.3$. No se encontró pacientes con TSVC menores de 15 días de edad. El 43\% de los pacientes $(n=6)$ tenían entre 16 y 30 días de vida al presentar el evento de TSVC y otro $43 \%$ de los pacientes tenían entre 46 y 56 días de vida, $14 \%$ de los niños $(n=2)$ tenían entre 31 y 45 días de vida.

7 (50\%) pacientes tenían el antecedente de infección respiratoria o gastrointestinal reciente o presente al momento de su ingreso.

La presentación clínica del cuadro de TSVC se caracterizó por convulsiones en 12 pacientes (86\%), deshidratados 9 (64\%) y 14 (100\%) presentaron fontanela anterior tensa.

Todos los pacientes presentaron anemia con niveles de $\mathrm{Hb}$ promedio de $6.7 \mathrm{~g} / \mathrm{dl}$. El promedio del recuento de leucocitos fue de 9,900. (Ver tabla No.1) Tres pacientes se presentaron con leucocitosis $(21.4 \%)$, un paciente tenía trombocitopenia (7\%), y uno trombocitosis (7\%). 
Tabla No.1: Estudios de gabinete en pacientes con TSVC.

\begin{tabular}{lcc}
\hline \multicolumn{1}{c}{ Características } & $\begin{array}{c}\text { Valor } \\
\text { promedio }\end{array}$ & De $^{*}$ \\
\hline $\begin{array}{l}\text { Leucocitos } \\
\text { (cel/ml) }\end{array}$ & 9,900 & $\pm 3,836$ \\
$\begin{array}{l}\text { Hemoglobina } \\
\text { (g/dL) }\end{array}$ & 6.7 & \pm 2.1 \\
$\begin{array}{l}\text { Plaquetas } \\
\text { (pla/ml) }\end{array}$ & 282,571 & $\pm 134,607$ \\
$\begin{array}{l}\text { Número de senos } \\
\text { afectados }\end{array}$ & 3.4286 & \pm 1.3 \\
\hline
\end{tabular}

Fuente: Instrumento TSVC en emergencia pediátrica *DE: Desviación estándar

Tres pacientes fallecieron (21.4\%) durante las primeras 72 horas de estancia hospitalaria. A estos se encontró con niveles de hemoglobina bajos $(6.8,4.1$ y $1.6 \mathrm{mg} / \mathrm{dl})$ y recuentos variables de leucocitos $(7,400,8,400$ y $13,700 \mathrm{cel} / \mathrm{ml})$ y plaquetas $(187,000,39,000$ y $533,000 \mathrm{pla} / \mathrm{ml})$.

Al revisar las tomografías iniciales se encontró que el Seno Sagital Superior estuvo afectado en todos los pacientes ( $n=14,100 \%)$, seguido por los senos laterales izquierdo $(n=11,78.6 \%)$ y lateral derecho $(n=10,71.4 \%)$. El seno recto se vió afectado en 7 pacientes (50\%), el seno sigmoideo derecho e izquierdo en 3 casos (21.4) cada uno. 13 pacientes (92.8\%), tuvieron trombosis en más de un seno venoso. En los pacientes que fallecieron, dos tenían los 6 senos afectados y uno 4 senos. (Ver tabla No. 2).

Todos los pacientes fueron manejados en forma médica con antibióticos por cuadro infeccioso, corrección de la deshidratación y de la anemia. Tres pacientes fueron sometidos a cirugía, dos para drenaje de hematoma más craniectomía descompresiva y uno para drenaje de hematoma subdural por trépano. Ningún paciente recibió anticoagulantes.

El seguimiento a los sobrevivientes en consulta externa se realizó durante un promedio de 10.7 meses ( \pm 8 meses), con un rango entre 6-30 meses y una moda de 6 meses. En la Tabla No. 2 se muestran las secuelas en relación con el número de senos afectados. Se encontró que el $50 \%(n=7)$ del total tenía retraso psicomotor severo y $29 \%(n=4)$ presentó desarrollo normal.

Los estudios tomográficos con las características más relevantes se muestran en las Imágenes de las Figuras 1-5. En las figuras 1-4 se presentan las secuencias tomográficas de 4 pacientes sobrevivientes con retraso psicomotor severo, se muestran las imagenes tomográficas al momento del diagnóstico, donde se identifica las zonas hiperdensas de trombosis venosa y también se muestra las secuencias de controles tomográficos hasta el momento del alta.

\section{Tabla No. 2: Secuelas según el número de senos afectados.}

\begin{tabular}{|c|c|c|c|c|c|c|}
\hline \multirow{2}{*}{ 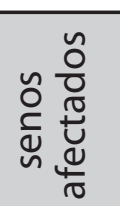 } & \multicolumn{2}{|c|}{ fallecieron } & \multicolumn{2}{|c|}{$\begin{array}{c}\text { retraso } \\
\text { psicomotor }\end{array}$} & \multicolumn{2}{|c|}{$\begin{array}{c}\text { desarrollo } \\
\text { normal }\end{array}$} \\
\hline & $\mathrm{n}$ & $\%$ & $\mathrm{n}$ & $\%$ & $\mathrm{n}$ & $\%$ \\
\hline 1 & 0 & 0 & 1 & 7 & 0 & 0 \\
\hline 2 & 0 & 0 & 0 & 0 & 1 & 7 \\
\hline 3 & 0 & 0 & 5 & 36 & 2 & 14 \\
\hline 4 & 1 & 7 & 1 & 7 & 1 & 7 \\
\hline 6 & 2 & 14 & 0 & 0 & 0 & 0 \\
\hline Total & 3 & 21 & 7 & 50 & 4 & 28 \\
\hline
\end{tabular}

Fuente: Instrumento TSVC en emergencia pediátrica

Como se observan en las figuras, se encontraron lesiones parenquimatosas asociadas a la TSVC. Estos incluyen áreas focales de edema, trombosis venosa cerebral, infarto venoso cerebral hemorrágico, edema cerebral difuso, y hemorragia subaracnoidea aislada. En la figura 5 se muestran lesiones cerebrales encontradas asociadas a la trombosis venosa cerebral como lo son el infarto cerebral hemorrágico y los hematomas. 


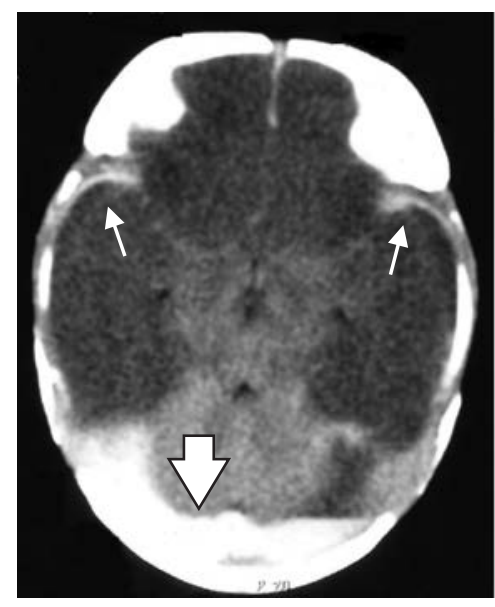

A

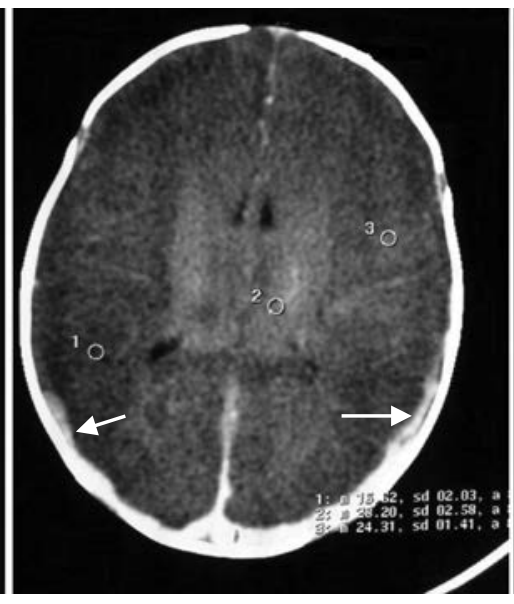

B

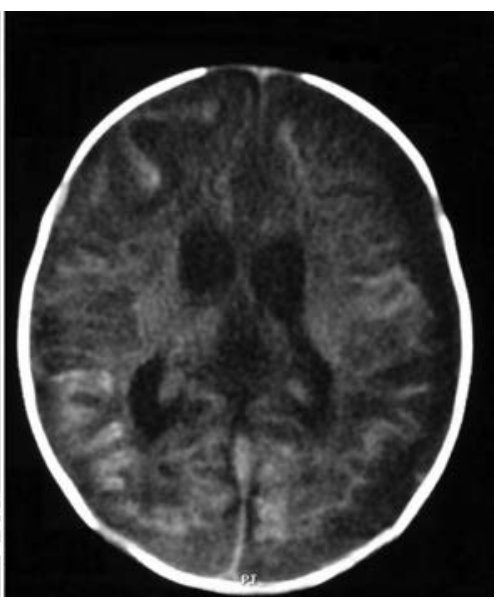

C

Figura 1. En la imagen $A$ se observa trombosis de los senos laterales como una zona hiperdensa señalada por la flecha blanca y trombosis de los senos esfenoparietales (flechas blancas delgadas). B hematoma subdural laminar bilateral (flechas largas) y la medición de la densidad cerebral de $16 \cup$ Hounsfield. C tomografía tomada un mes después que muestra

daño cerebral y aumento compensatorio del tamaño de los ventrículos laterales. bilateral (flechas largas) y la medición de la densidad cerebral de 16 U Hounsfield. C tomografía tomada un mes después que muestra daño cerebral y aumento compensatorio del tamaño de los ventrículos laterales.

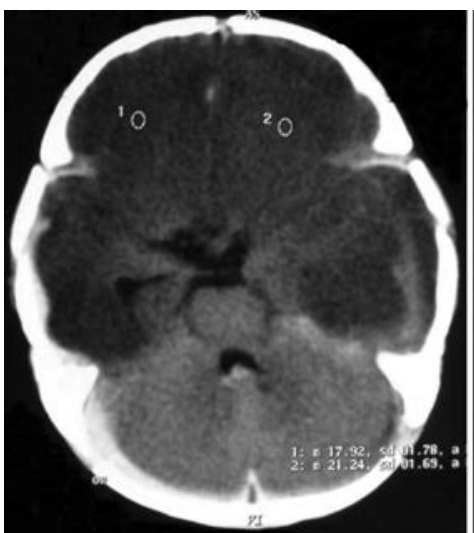

A

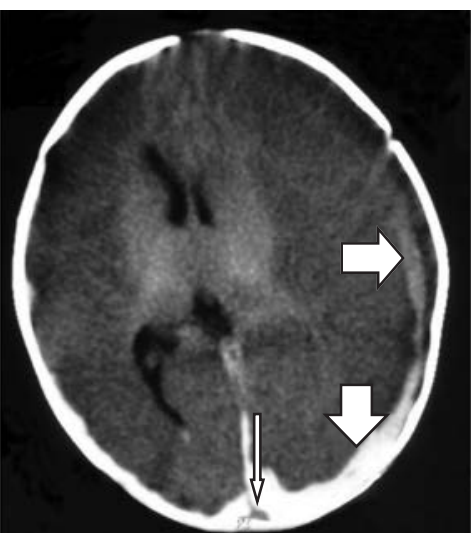

B

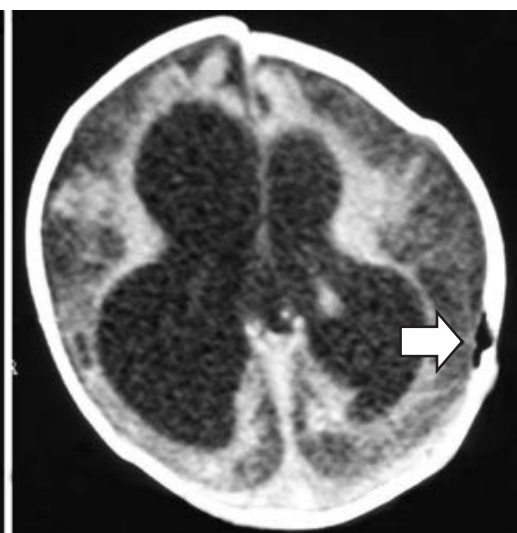

C
Figura 2. En la imagen A se aprecia la hipodensidad de los hemisferios cerebrales, medida en 17 y 21 unidades Hounsfield. En la imagen B se observa un hematoma subdural laminar izquierdo (flechas gruesas), desplazamiento cerebral hacia el lado derecho y el signo delta vacio (flecha delgada). La imagen $C$ es obtenida dos meses después, se observa la gran dilatación ventricular por pérdida de tejido cerebral, el cabalgamiento óseo de la sutura coronal derecha y sagital, y el agujero de trépano quirúrgico señalado con la flecha. 


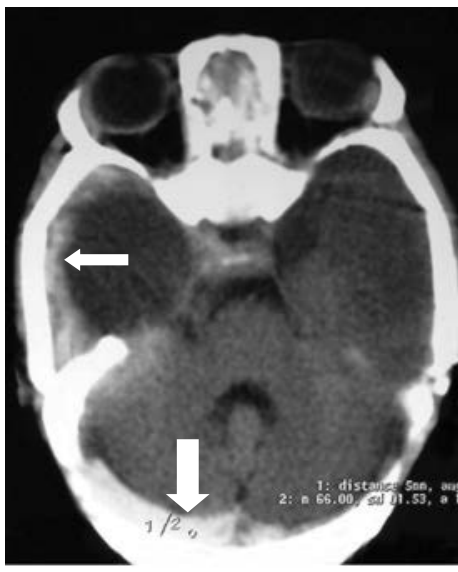

A

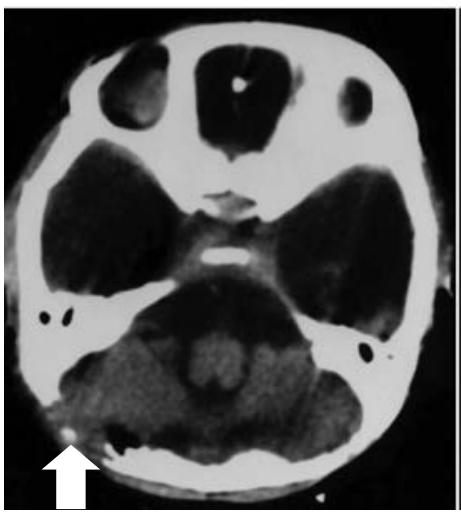

$\mathrm{D}$

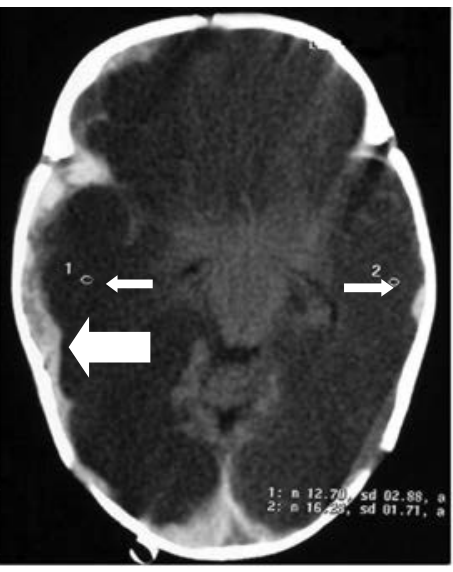

B

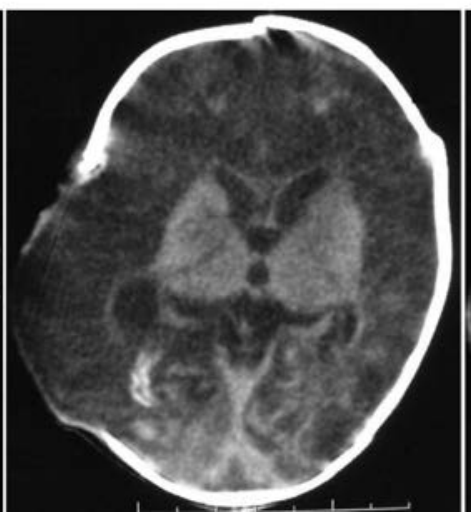

E

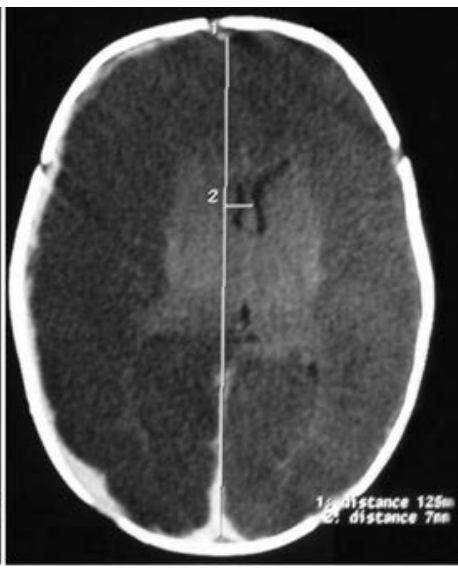

C

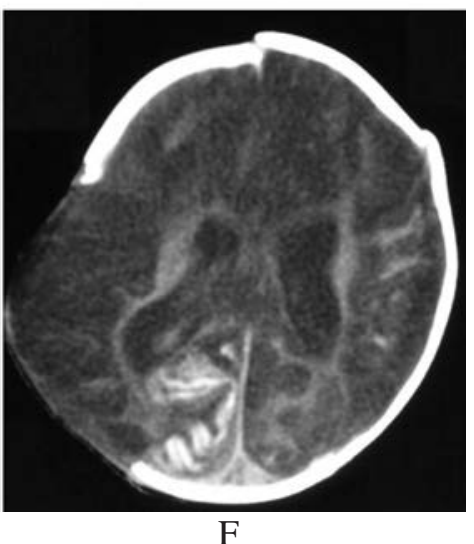

Figura 3. En la imagen A se observa un hematoma subdural laminar temporal derecho (flecha delgada) e hipodensidad del lóbulo temporal derecho, el seno lateral derecho con trombosis de $5 \mathrm{~mm}$ y con densidad de 66 unidades Hounsfield (señalado con flecha gruesa). Imagen B con hematoma subdural laminar derecho (flecha gruesa) y medición de densidad cerebral de 12 unidades Hounsfield (flechas delgadas). Imagen C desplazamiento cerebral hacia la izquierda de $7 \mathrm{~mm}$. Imágenes $\mathrm{D}$, $\mathrm{E}$, y $\mathrm{F}$ de tomografía realizada 15 días después. En la imagen D la flecha blanca indica el sitio de la craniectomía y en las imágenes $E$ y $F$ se observa la amplia craniectomía derecha con herniación cerebral, la presencia de hipodensidad cerebral y aumento del tamaño ventricular como consecuencia del daño cerebral. Puede también observarse el cabalgamiento de las suturas coronal izquierda y sagital. 


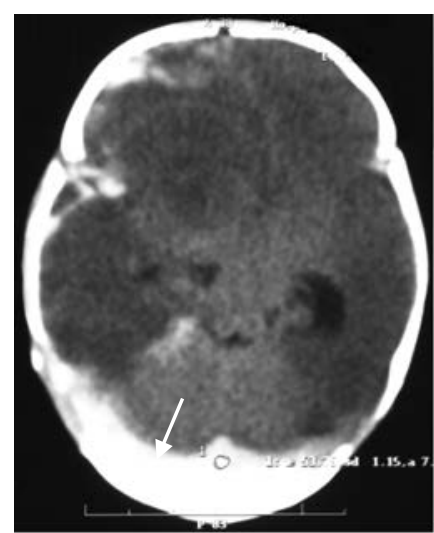

A

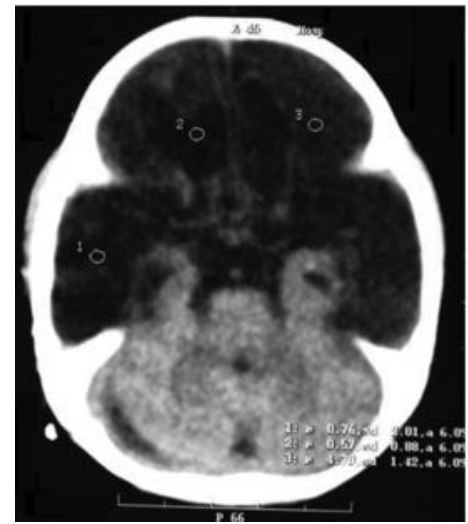

$\mathrm{D}$

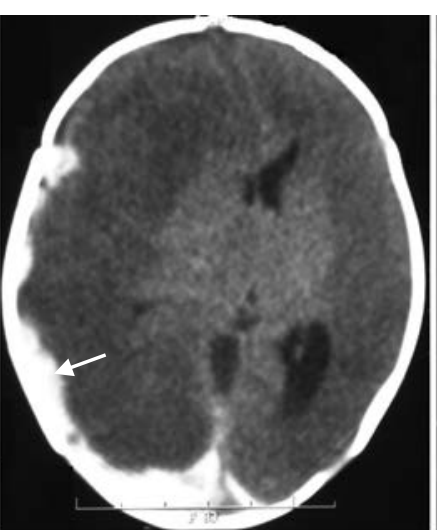

B

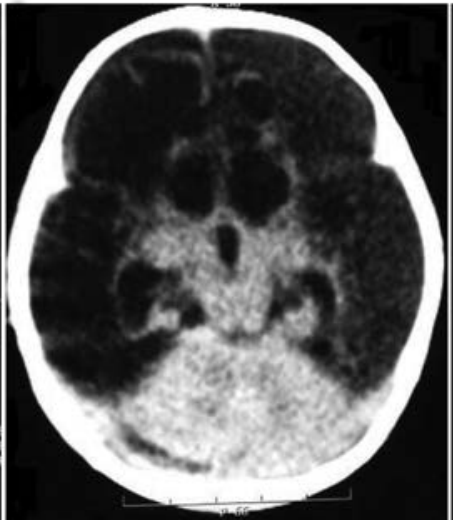

E

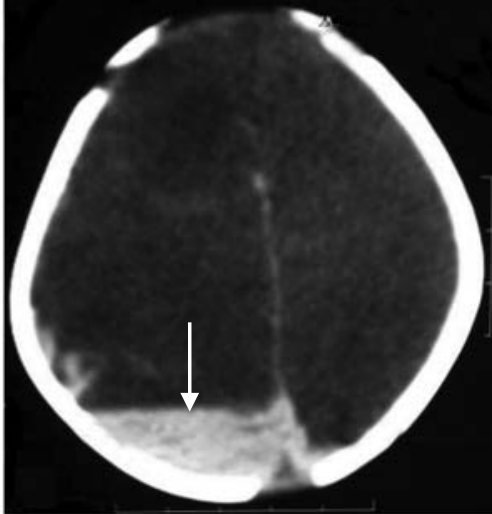

C

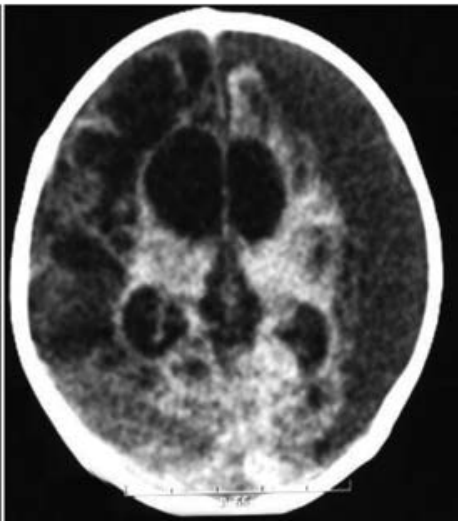

$\mathrm{F}$
Figura 4. En la imagen A se observa la trombosis de ambos senos laterales e hipodensidad cerebral generalizada. En la imagen $B$ y $C$ se observa hematoma subdural (flecha negra) y mayor edema en hemisferio derecho. En la imagen D, E y F se observan Tomografía control obtenida 40 días después del caso en la cual se observa integridad imagenológica de estructuras de la fosa posterior (cerebelo y tallo cerebral) pero daño de todos los hemisferios cerebrales con aumento compensatorio del tamaño de los ventrículos laterales.

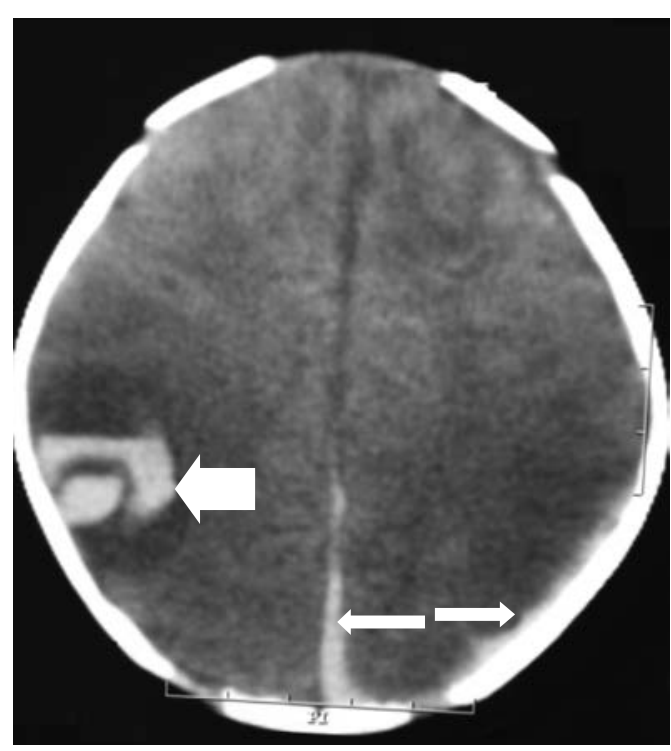

Figura 5. Imagen corresponde a lesiones adicionales a la TSVC encontradas en uno de los pacientes mostrando un infarto hemorrágico (flecha gruesa) y la presencia de hematoma subdural (flechas delgadas). 


\section{DISCUSION}

La incidencia anual de TSVC es de 0.67 casos por 100,000 niños, de los cuales la mitad de los casos se presenta en neonatos, por lo que en esta población se alcanza una incidencia de 2.6 a 12 casos por 100,000 neonatos. ${ }^{(1-4)}$ deVeber $y$ colaboradores reportaron 160 casos de TSVC en Canada en el período de 1996 al 2001. De estos el $54 \%$ eran menores de un año y el $43 \%$ de los casos eran neonatos. ${ }^{(4)}$ En este estudio 6 pacientes (43\%) eran neonatos, menores de 28 días, y el resto, 8 pacientes (57\%) menores de 2 meses. No se tuvo ningún paciente mayor de 2 meses.

Aunque hay pocos estudios de población menor de 2 meses se debe considerar como una población especial propensa a desarrollar esta patología. ${ }^{(5-7)}$ Los síntomas más frecuentemente observados en los pacientes que sufren TSVC son irritabilidad, somnolencia, letargia, disminución en la fuerza de succión, convulsiones, paresias, signos de hipertensión endocraneana como abombamiento de la fontanela anterior, vómito, hipotonía y distres respiratorio. ${ }^{(5,8-9)}$

El diagnóstico puede alcanzar un cierto grado de dificultad por la sintomatología inespecífica, la similitud clínica a un cuadro de encefalitis o meningitis, y por la falta de sospecha clínica sobre ésta patología vascular cerebral. ${ }^{(3,6,9)}$ Los hallazgos clínicos encontrados fueron fontanela anterior tensa (100\%), convulsiones (86\%), deshidratación (50\%). Una de las limitaciones encontradas fue que no se registró la escala de Glasgow al ingreso o la presencia de otros síntomas distintos a los ya descritos.

Dentro de los factores asociados al desarrollo de TSVC se han reportado infección, deshidratación, trauma, mala nutrición, alteraciones hematológicas como anemia con o sin microcitosis, deficiencia de hierro, trombocitosis, niveles elevados de factor VIII, $\beta$-talasemia, mutación del factor $V$ de Leiden, mutación 20210A de la protrombina, y otros factores protrombó- ticos. En población pediátrica mayor se ha relacionado además con enfermedades congénitas cardíacas cianóticas, mastoiditis, falla renal, cáncer, diabetes, celulitis orbitaria preseptal y otras enfermedades crónicas. ${ }^{(7,10-13)}$ Por limitaciones de laboratorio en ninguno de los pacientes se realizó estudios especiales de factores de riesgos protrombóticos.

El diagnóstico se basa en estudios de neuroimagen, y dentro de estos el más rápido, barato y más frecuentemente utilizado es la Tomografía Computarizada de cerebro, no obstante debe existir un alto grado de sospecha clínica para realizar cortes y reconstrucciones que faciliten la visualización de los senos venosos. ${ }^{(10)}$ La tomografía simple tiene una sensibilidad y especificidad de 64.6 y $97.2 \%$ respectivamente para detectar la TSVC. ${ }^{(11)}$ Hasmi et al reportan un rango de error de 10 a $26 \%$ en las tomografías simples. ${ }^{(2)}$ El estudio con mayor rango de sensibilidad y especificidad es la Resonancia Magnética, pero debe considerarse el tiempo de evolución de la trombosis porque las señales de la trombosis venosa van a variar con la edad del trombo. Algunas veces será necesaria la realización de tomografía con medio de contraste para visualizar los senos venosos o angiotomografía en fase venosa. La venografía por Resonancia Magnética puede realizarse sin medio de contraste en la fase Time Of Flight (TOF) o con la administración de medio de contraste. ${ }^{(10,13-16)}$ En los resultados presentados, el diagnóstico se estableció por tomografía simple, en ningún paciente se realizó reconstrucción o administración de medio de contraste para realizar la fase venosa, ni estudios por Resonancia Magnética.

El tratamiento de la TSVC dependerá del factor desencadenante o predisponente, del tiempo de evolución y del estado del paciente al momento de su evaluación. En el estudio de DeVeber et al, Johnson et al y Bames et al, citados por Sébire en su publicación del 2005, se demostró que los pacientes tratados con anticoagulantes tenían menor mortalidad, lo 
que dio un respaldo para utilizar la anticoagulación en neonatos. ${ }^{(1)}$ No obstante aún no se cuenta con suficiente evidencia para recomendar su uso rutinario. ${ }^{(3,6-9)}$ Los estados de alteración hidro-electrolítica deberán ser corregidos y la infección, de existir, deberá ser tratada. El $100 \%$ de los pacientes tenían anemia que requirió transfusión y ninguno recibió terapia anticoagulante.

Se encontró una mortalidad de $21 \%$, superior a la reportada en otros estudios (6 a 19\%), ${ }^{(1-9)}$ y esto puede deberse a que los pacientes fueron aquellos que por el edema cerebral y hematomas subdurales se presentaron al Servicio de
Neurocirugía, lo que representa una subpoblación con mayor compromiso neurológico. Un $50 \%$ del total de los pacientes tuvo secuelas neurológicas y sólo el $28.6 \%$ tuvo un desarrollo normal.

La TSVC es una entidad vascular con alta tasa de mortalidad y de severas secuelas neurológicas, por lo que la comunidad pediátrica debe reconocerla en sus etapas tempranas para establecer un pronto tratamiento y evitar, en lo posible, sus devastadoras consecuencias. Es recomendable realizar estudios más controlados de esta patología a nivel nacional para poder establecer protocolos de manejo.

\section{BIBLIOGRAFÍA}

1. Sébire G, Tabarki B, Saunders E, Leroy I, Liesner R, Saint-Martin C, et al. Cerebral venous sinus thrombosis in children: risk factors, presentation, diagnosis and outcome. Brain 2005; 128: 477-489. DOI: http://dx.doi.org/ 10.1093/brain/awh412.

2. Hashmi M, Wasay M. Caring for cerebralvenoussinusthrombosis in children. J Emerg Trauma Shock. 2011; 4(3): 389-94. DOI: $10.4103 / 0974-2700.83870$

3. Saxonhouse MA. Thrombosis in the Neonatal Intensive Care Unit.ClinPerinatol. 2015; 42(3): 651-73. DOI: 10.1016/j.clp.2015.04. 010.

4. deVeber G, Andrew M, Adams C, Bjornson B, Booth F, Buckley DJ Cerebral sinovenous thrombosis in children. N Engl J Med [Revista en internet]. 2001 [citado 15 Mar 2016]; 345(6):417. Disponible en: http://www. nejm.org/doi/pdf/10.1056/NEJM20010809 3450604.

5. Berfelo FJ, Kersbergen $\mathrm{KJ}$, van Omen $\mathrm{CH}$, Govaert P, van Straaten HL, Poll-The BT, et al. Neonatal cerebralsinovenousthrombosis from symptom to outcome. Stroke. 2010; 41(7): 1382-8. DOI: 10.1161/STROKEAHA. 110.583542 .

6. Moharir MD, Shroff $M$, Pontigon AM, Askalan R, Yau I, Macgregor D, et. Al. A prospective outcome study of neonatal cerebralsinovenousthrombosis. J Child Neurol2011; 26(9): 1137-44. DOI: 10.1177/ 0883073811408094.

7. Kenet $G$, Lütkhoff L, Albisetti M, Bernard T, Bonduel $M$, Brandao $L$, et al. Impact of Thrombophilia on Risk of Arterial Ischemic Stroke or Cerebral Sinovenous Thrombosis in Neonates and Children: A Systematic Review and Meta-Analysis of Observational Studies.Circulation[Revista en internet], 2010[citado el 03 Nov 2015]; 121; 18381847. Disponible http://circ.ahajournals. org/cgi/content/full/121/16/1838.

8. Torres VM, Saddi VA. Systematic review: hereditary thrombophilia associated to pediatric strokes and cerebral palsy. J Pediatr. (Rio J) 2015; 91(1): 22-29. DOI: 10.1016/ j.jped.2014.08.004. 
9. Fitzgerald K, Williams L, Garg B, Carvalho K, Golomb M. Cerebral sinovenous thrombosis in the neonate. Arch Neurol. 2006; 63: 405-409. DOI: 10.1001/archneur.63.3.405.

10. Romero A, Marco P, Verdú J, Sanchez S, Castaño V. Trombofilia Genética y Trombosis de Senos Venosos Cerebrales. MedClin (Barc) 2007; 128(17): 655-656. DOI: 10. $1157 / 13102055$

11. Buyck PJ, De Keyzer F, Vanneste D, Wilms G, Thijs V, Demaerel P.CT density measurement and $\mathrm{H}: \mathrm{H}$ ratio are useful in diagnosing acute cerebralvenoussinusthrombosis. AJNR Am J Neuroradiol 2013; 34(8): 1568-72. DOI: 10. 3174/ajnr.A3469.

12. Menascu S, Lotan A, Ben Zeev B, Nowak-Gottl U, Kenet G. Cerebralvenous thrombosis in the mediterranean area in children. Mediterr J Hamatol Infect Dis 2011;3(1): e2011029. doi: 10.4084/MJHID.2011.029.

13. Abarca J, Botella C, Concepción L, Cortés J, Gallego J, Ballenilla F. Hemorragia intracerebral secundaria a trombosis espontanea de una anomalía venosa del desarrollo: 2 casos y revisión de la literatura. Neurocirugía 2009; 20:265-271.DOI: 10.1016/S1130-1473 (09)70166-6

14. Yun JH, Ko JH, Lee MJ. Early Spontaneous Recanalization of Sigmoid Sinus Thrombosis Following a Closed Head Injury in a Pediatric Patient: A Case Report and Review of Literature. J Korean NeurosurgSoc. 2015; 58(2):150-4. DOI: 10.3340/jkns.2015.58.2. 150.

15. Russi ME, González V, Campistol J. Cerebral venous thrombosis in the pediatric age: clinical presentation, risk factors, diagnosis and treatment. RevNeurol [Revista en internet] 2010 [citado el 03 oct 2015]; 51(11): 661-8. Disponible en: http://www.revne urol.com/sec/resumen.php?or=pubme d\&id=2010355\#

16. Dlamini N, Billinghurst L, Kirkham FJ. Cerebral venous sinus (sinovenous) thrombosis in children. NeurosurgClin N Am. 2010; 21(3): 511-27. DOI: 10.1016/j.nec.2010. 03.006 . 